\title{
Long-Term Efficacy and Safety of Subconjunctival/ Perilesional 5-Fluorouracil Injections for Ocular Surface Squamous Neoplasia
}

This article was published in the following Dove Press journal:

Drug Design, Development and Therapy

\section{Yuzhao Sun \\ Rui Hua (D)}

Department of Ophthalmology, The First Affiliated Hospital of China Medical University, Shenyang, Liaoning II000I, People's Republic of China
Correspondence: Rui Hua

Department of Ophthalmology, The First Affiliated Hospital of China Medical University, No. 155 Nanjingbei Street, Heping District, Shenyang, Liaoning I I000I, People's Republic of China

Tel +86- I 3-840583355

Fax +86-24-83282630

Email woodshua@I26.com
Purpose: To investigate the effectiveness and safety of subconjunctival/perilesional 5-fluorouracil injections on ocular surface squamous neoplasia (OSSN) during a 3-year follow-up period.

Patients and Methods: We followed up six patients with intraepithelial OSSN (in one eye each) that had regressed after subconjunctival/perilesional 5-fluorouracil injections. Conjunctival fluorescein angiography (FA) and indocyanine green angiography (ICGA), as well as anterior segment optical coherence tomography (AS-OCT), were performed to evaluate the OSSN status 3 years after initiation of treatment.

Results: The mean age of patients (five males, one female) at baseline was $62.3 \pm 11.6$ years. The mean number of 5-fluorouracil injections was $17.0 \pm 8.6$, with a mean treatment duration of 13.0 \pm 7.4 weeks. At the final visit, both intratumoral and conjunctival feeding vessels had disappeared on ICGA and FA, with no neovascularization-related leakage, in accordance with the results of AS-OCT. The period from complete tumor regression to final visit according to AS-OCT was $32.5 \pm 4.2$ months, which was longer than that according to ICGA (31.3 \pm 3.2 months, $\mathrm{p}=0.034)$. The final best-corrected visual acuity was similar to that at baseline $(\mathrm{p}=0.128)$. No side effects were observed in any of the eyes.

Conclusion: Subconjunctival/perilesional 5-fluorouracil injections are an effective and safe treatment for OSSN. Future studies with a larger sample size are warranted for confirmation of our findings, as well as investigation into the reasons for residual areas of non-perfusion in the conjunctiva.

Keywords: ocular surface squamous neoplasia, subconjunctival injection, 5-fluorouracil, long-term follow-up, conjunctival angiography, optical coherence tomography

\section{Introduction}

Lee et $\mathrm{al}^{1}$ first coined the term "ocular surface squamous neoplasia" (OSSN) to describe an entity that progresses from primary dysplastic to invasive carcinomatous lesions in the corneal or conjunctival epithelium ${ }^{2}$ and can potentially become life threatening. ${ }^{3}$ Histopathology is considered the gold standard for its diagnosis. ${ }^{4}$ Nowadays, in vivo confocal microscopy is used to monitor OSSN during topical treatment. ${ }^{5}$ Multispectral autofluorescence imaging can also be used to detect OSSN lesions, ${ }^{6}$ and anterior segment optical coherence tomography (AS-OCT) can provide an "optical biopsy," assisting in the diagnosis and treatment of OSSN lesions.

In addition to traditional AS-OCT, we previously reported the angiographic characteristics of OSSN by conjunctival fluorescein angiography (FA) and 
indocyanine green angiography (ICGA) for the first time. ${ }^{8}$ Moreover, we discovered that conjunctival angiography could visualize residual tumor-related vessels of treated OSSN, even where AS-OCT revealed no lesions. ${ }^{8}$ Correspondingly, Liu et $\mathrm{al}^{9}$ investigated OCTangiographic characteristics of OSSN and concluded that such imaging permitted the demonstration and enumeration of vessel structure within, beneath, and surrounding OSSN.

Treatments for OSSN include topical chemotherapy, cryotherapy, and excision. Recently, Theotoka et $\mathrm{al}^{10}$ reported a case of spontaneous regression of OSSN. Topical 5-fluorouracil has proved to be a safe and effective treatment for preinvasive OSSN and 50\% of invasive OSSN in the long term. ${ }^{11}$ However, although 5-fluorouracil has an efficacy of $85-100 \%$ as primary treatment for OSSN, it has also exhibited a $1.1-43 \%$ tumor recurrence rate. $^{12}$

We previously demonstrated that subconjunctival/perilesional 5-fluorouracil injection was an effective anatomic and functional treatment for OSSN in the short term, delivering 5-fluorouracil to the tumor site directly, leading to rapid tumor resolution. ${ }^{8}$ In the present study, we aimed to investigate the long-term effectiveness and safety of subconjunctival/perilesional 5-fluorouracil injections for treatment of OSSN, based on both conjunctival angiography and AS-OCT performed 3 years after initiation of treatment.

\section{Patients and Methods}

In this retrospective study, we reevaluated one eye each of six intraepithelial OSSN patients (five males and one female), 3 years after initiation of treatment with three phase subconjunctival/perilesional 5-fluorouracil injections. $^{8}$ Surrogate markers of intraepithelial OSSN are a clear plane of separation and increased thickness visible on AS-OCT. ${ }^{13}$ After three phases of treatment, the tumors completely subsided under slit lamp examination. At that review, we also performed AS-OCT, FA and ICGA examinations for each patient to confirm that the epithelium returned to a normal shape and that intratumoral vessels, conjunctival feeding vessels, and corneal neovascularization disappeared. If a tumor recurred, we returned to the second phase of treatment.

We calculated patients' best-corrected visual acuity (BCVA) in logarithm of the minimum angle of resolution $(\log M A R)$ form and performed anterior segment digital photography (MediWorks, Shanghai, China), AS-OCT
(Spectralis OCT2; Heidelberg Engineering, Heidelberg, Germany). We also performed simultaneous conjunctival ICGA and FA in stereo pair mode (Spectralis HRA+OCT; Heidelberg Engineering, Heidelberg, Germany) to assess the progression of OSSN and residual vascular tissue. All six patients also underwent systematic examination, and those suspected to have metastasis to lymph nodes or other tissues or organs were excluded from the study, but no patient was excluded.

Statistical analyses were performed using IBM SPSS Statistics for Windows (version 19.0; IBM Corp., Armonk, NY, USA). The data are expressed as mean \pm standard deviation. The logMAR BCVA at baseline was comparable to that 3 years after initiation of 5 -fluorouracil therapy, using two-tailed, paired t tests. A p value $<0.05$ was considered statistically significant.

\section{Results}

The mean age of patients at baseline was $62.3 \pm 11.6$ years (Table 1). The mean number of 5-fluorouracil injections over the 3-year follow-up period was 17.0 \pm 8.6 with a mean treatment duration of $13.0 \pm 7.4$ weeks. Patients 1 and 4, who exhibited a focal pattern of intratumoral vessels, received 22 and 10 injections, respectively, to achieve complete anatomic regression of OSSN. Patients 5 and 6, who exhibited sea fan-shaped intratumoral vessels, received 9 and 10 injections, respectively. Vitreous hemorrhage secondary to diabetic retinopathy, and branch retinal vein occlusion (BRVO)-related macular edema, were observed in patients 1 and 2, respectively, at their final visit. The final $\log$ MAR BCVA was $0.38 \pm 0.53,3$ years after initiation of treatment, which was similar to that at baseline $(0.69 \pm 0.66)(\mathrm{t}=1.824, \mathrm{p}=0.128)$.

At baseline, conjunctival ICGA revealed conjunctival feeding vessels in four eyes, including two eyes with a focal pattern of intratumoral vessels and two eyes with sea fanshaped intratumoral vessels (Table 1). At their final visit, intratumoral and conjunctival feeding vessels had disappeared (as revealed upon ICGA) from all six eyes, although areas of non-perfusion remained deep in the conjunctiva. No leakage related to neovascularization of OSSN was observed upon FA for any of the patients. Similarly, ASOCT revealed a normal structure of the cornea and conjunctiva in the original OSSN location of all six eyes (Figures 1-3). However, the period from complete tumor regression to final visit according to AS-OCT was $32.5 \pm 4.2$ months, which was longer than that according to ICGA (31.3 \pm 3.2 months, $\mathrm{t}=2.907, \mathrm{p}=0.034)$; with a mean 


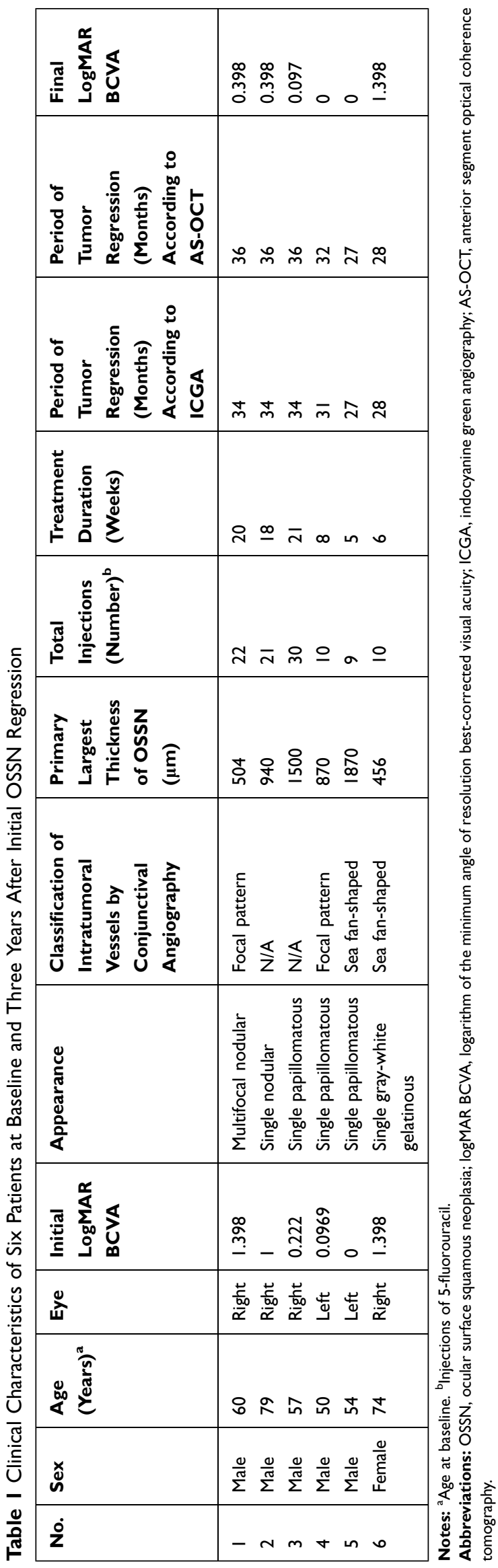

difference of $1.2 \pm 1.0$ months. No side effects were observed in any of the six eyes at the final visit.

\section{Discussion}

We assessed the long-term effectiveness and safety of subconjunctival/perilesional 5-fluorouracil injections for the treatment of OSSN. Follow-up is of the utmost importance to verify the effectiveness of therapy because OSSN may relapse years after initial treatment. Joag et al, ${ }^{14}$ while evaluating topical 5-fluorouracil treatment of OSSN, reported recurrence rates of $6 \%$ and $15 \%$ at 1 and 2 years, respectively. A report from Kenya noted a 1-year recurrence rate of $11 \%$ in patients being treated with fluorouracil. ${ }^{15}$ Importantly, we demonstrated that there was no relapse of OSSN and there were no side effects 3 years after initiation of treatment.

Although surgical removal is widely used to treat OSSN that affects less than 4 clock hours of limbus, sole topical chemotherapy is proven to achieve complete tumor resolution ${ }^{16}$ with less injury. Common topical agents include mitomycin $\mathrm{C}$, alpha interferon (IFN $\alpha$ )-2b, and 5-Fluorouracil. Among topical agents, mitomycin $\mathrm{C}$ has more frequent and severe adverse effects than IFN $\alpha-2 b$ or 5-Fluorouracil, including pain, epitheliopathy, allergic conjunctivitis, hyperemia, ectropion, punctal stenosis, ${ }^{17}$ and limbal stem cell deficiency. ${ }^{18}$ Topical IFN $\alpha-2 b$ shows favorable outcomes in the treatment of both primary and recurrent OSSN, although with a longer duration of treatment than is required with mitomycin C. ${ }^{19}$ Topical IFNa$2 b$ is off-label and unavailable in China. The main sideeffect associated with sub-conjunctival IFN $\alpha-2 b$ injections is a flu-like syndrome that lasts for approximately 48 $\mathrm{h}$ after administration of the injection. ${ }^{20} 5$-fluorouracil has several theoretical advantages over mitomycin $\mathrm{C}$ and interferon alpha- $2 \mathrm{~b}$, including a lower cost of treatment, no requirement for refrigeration, and less frequent dosing regimens. ${ }^{21}$ According to real-world evidence, the efficacy of topical chemotherapy is lower in large or invasive OSSN because topical agents are unlikely to reach the invasive cells. ${ }^{16}$ Thus, the advantages of this delivery mode include higher drug penetration, more direct delivery to the tumor site, faster tumor resolution, and assured compliance compared with topical 5-fluorouracil drops. ${ }^{8}$

During our 3-year follow-up, the average number of 5-fluorouracil injections was $17.0 \pm 8.6$, with a mean treatment duration of $13.0 \pm 7.4$ weeks. Venkateswaran et $\mathrm{al}^{22}$ discovered that time to resolution of OSSN after topical 5-fluorouracil administration was $6.6 \pm 4.5$ months. 


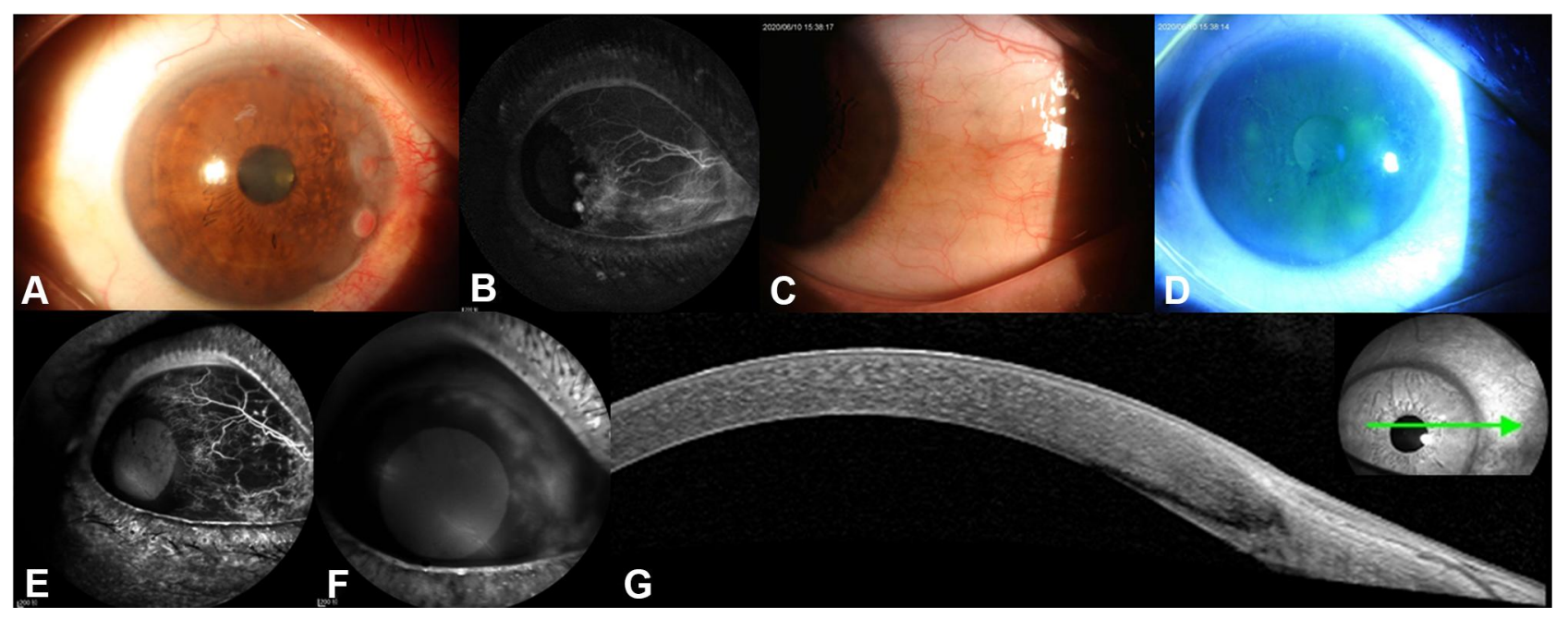

Figure I Three-year follow-up of case I, a 60-year-old male patient who had primary ocular surface squamous neoplasia in the right eye (A), with a focal pattern of intratumoral vessels ((B) indocyanine green angiography [ICGA]) at baseline. Anterior segment photography reveals that there is no tumor recurrence, only a small spot in the palpebral fissure at 3 o'clock (C), as well as rough corneal epithelium in the upper nasal quadrant, and scattered fluorescein staining dots (D). Early-phase ICGA visualizes a patch of non-perfusion in the palpebral fissure, with no intratumoral or conjunctival feeding vessels (E). Late-phase fluorescein angiography demonstrates corneal leakage (F), which is in accordance with the rough corneal epithelium and scattered fluorescein staining dots in (D) due to corneal injury by the patient. Anterior segment optical coherence tomography (AS-OCT; G) reveals a bright white tear-film layer overlying the cornea and conjunctiva, with a continuous thin corneal epithelium, without any high reflectance or abnormal thickening (green arrow indicates the direction of AS-OCT scanning).

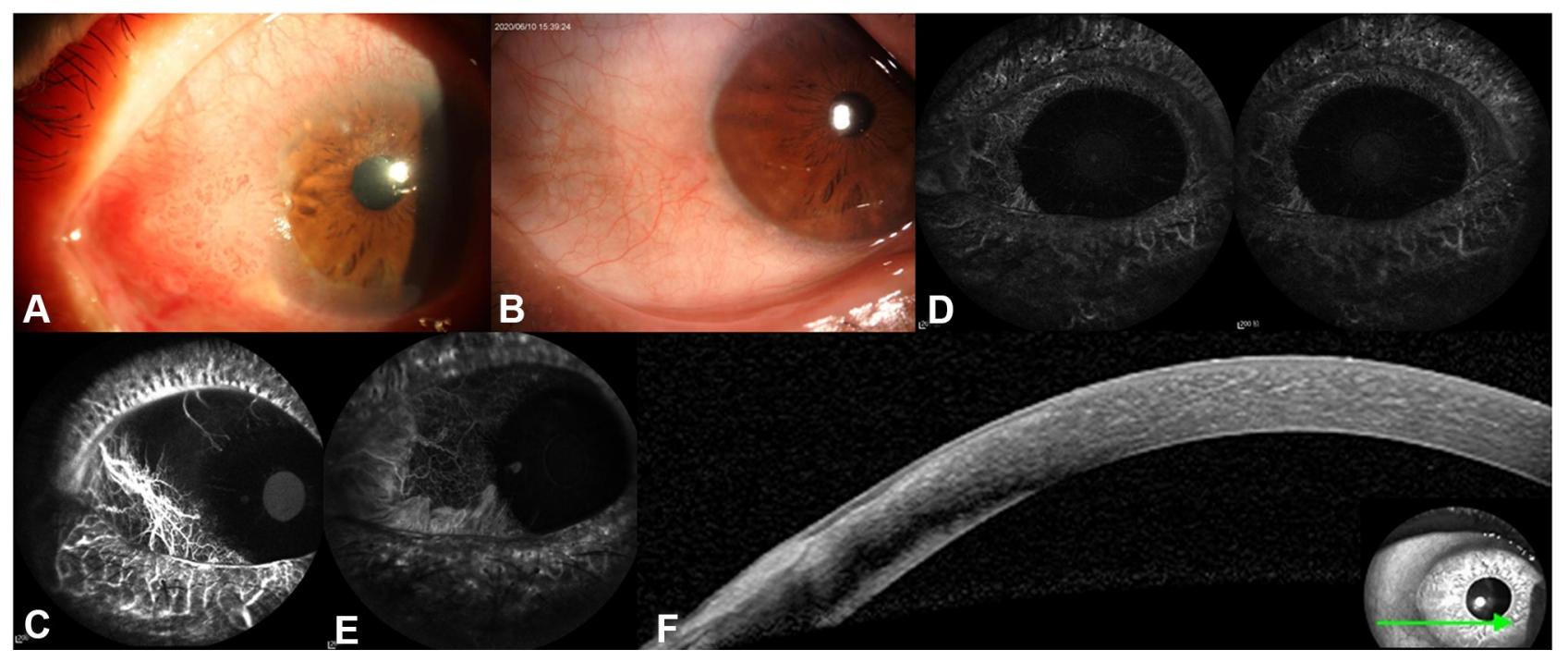

Figure 2 Three-year follow-up of case 3, a 57-year-old male patient with a primary diagnosis of ocular surface squamous neoplasia (OSSN) in the right eye at baseline (A). Anterior segment photography reveals a spot in the palpebral fissure between 8 and 9 o'clock, with a smooth and transparent cornea, and no recurrence of OSSN (B). Early-phase indocyanine green angiography (ICGA) visualizes a patch of non-perfusion in the palpebral fissure, with no intratumoral or conjunctival feeding vessels (C). Stereo pair-mode ICGA displaying a three-dimensional view of the entire anterior segment, with left and right polarization acquired in parallel (D). Late-phase ICGA also reveals the patch of non-perfusion (E). Anterior segment optical coherence tomography (AS-OCT; F) displays normal corneal structures at the original OSSN location (green arrow indicates the direction of AS-OCT scanning).

Parrozzani et al $^{11}$ reported that after a mean of 1.55 -fluorouracil courses, $83 \%$ of cases achieved complete tumor regression, statistically significantly related to a lack of fornix or tarsal involvement, tumor thickness $<1.5 \mathrm{~mm}$, and an absence of multifocality. From the current study, we can add that OSSN containing intratumoral vessels with a focal pattern needs more injections for complete anatomic regression than those containing sea fan-shaped intratumoral vessels.

At the final visit, ICGA and FA revealed the disappearance of both intratumoral and conjunctival feeding vessels with no neovascularization-related leakage secondary to OSSN in any of the six eyes, which is compatible with the results of AS-OCT. This indicated complete 


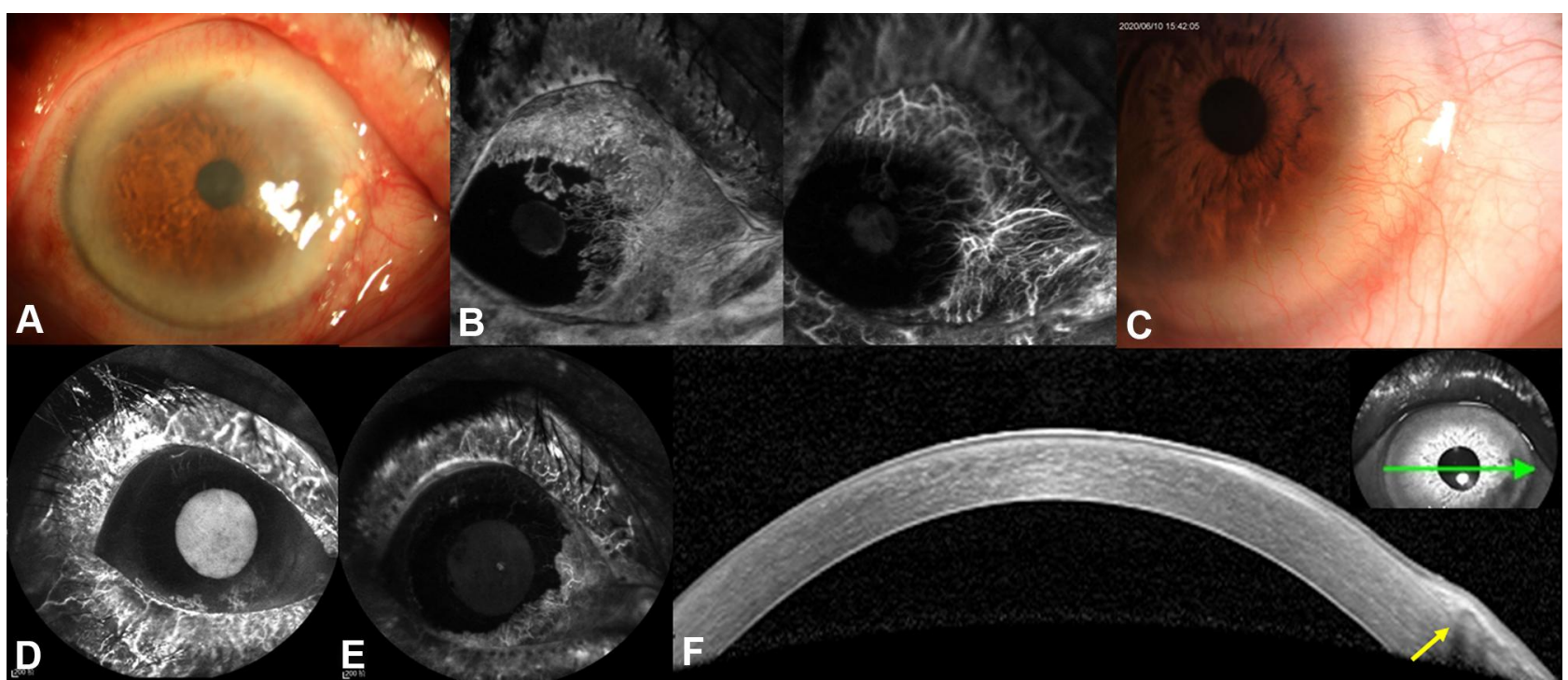

Figure 3 Three-year follow-up of case 6, a 74-year-old-female patient who had primary ocular surface squamous neoplasia in the right eye (A), with sea fan-shaped intratumoral vessels((B) left image: fluorescein angiography; right image: indocyanine green angiography [ICGA]), at baseline. Anterior segment photography (C) reveals that there is no recurrent mass on the corneal surface, and that a pterygium is growing into the corneal limbus between 3 and 6 o'clock. Early- and late-phase indocyanine green angiography reveals perfusion of new vessels in the lower nasal part of the corneal limbus, with no intratumoral or conjunctival feeding vessels (D and $\mathbf{E})$. Anterior segment optical coherence tomography (AS-OCT; F) reveals normal corneal structures and the pterygium growing into the corneal limbus between 3 and 6 o'clock (green arrow indicates the direction of AS-OCT scanning). The latter is visible as a thickened, highly reflective lesion between the corneal epithelium and Bowman's membrane in the subepithelial space (yellow arrow).

atrophy of intratumoral vessels and retention of the normal tight junctions of vascular endothelium. There was an approximately 16-week median delay between clinical and OCT resolution of OSSN, the longest delay being 29 weeks, ${ }^{23}$ indicating that AS-OCT may prevent the premature cessation of treatment. ${ }^{24}$ The period of complete tumor regression according to AS-OCT was $1.2 \pm 1.0$ months longer than that according to ICGA; therefore, judging tumor regression using only AS-OCT may increase the risk of recurrence.

Previously, in our short-term observational study, BCVA improved after treatment because of better anatomic outcomes, regression of intratumoral and conjunctival feeding vessels, or the reduced severity of side effects. ${ }^{8}$ Similarly, Chaugule et $\mathrm{al}^{25}$ reported that either 5-fluorouracil or interferon alpha- $2 \mathrm{~b}$ would guarantee stable or improved BCVA of giant OSSN eyes. However, our study showed that the final BCVA 3 years after initial OSSN treatment was comparable to that at baseline. This may be in part because of the vitreous hemorrhage and BRVO-related macular edema that occurred in patient 1 and patient 2, respectively.

Finally, stereo angiography was previously used in rats to visualize uveitis lesions in the retina and choroid in three dimensions. ${ }^{26}$ In our study, stereo ICGA was also used for the successful three-dimensional observation of eyes in which OSSN had regressed.
There are some limitations to this study, including a small cohort and retrospective design. Furthermore, we could not explain the residual area of non-perfusion deep in the conjunctiva in areas where primary OSSN had previously occurred. Further research is required to investigate the possible reasons for such non-perfusion.

\section{Conclusion}

In this study, subconjunctival/perilesional 5-fluorouracil injections are demonstrated to be effective and safe for treatment of OSSN in the long term ( 3 years). In addition, we have discovered that fewer injections may be required to achieve complete anatomic regression in those whose OSSN contains sea fan-shaped intratumoral vessels than in those with a focal pattern.

\section{Ethics Statement}

This study was approved by the Institutional Review Board of The First Affiliated Hospital of China Medical University, according to the Declaration of Helsinki. All patients provided written informed consent.

\section{Acknowledgments}

This manuscript is a sequel to our previous study, "Ocular surface squamous neoplasia: angiographic characteristics 
and response to subconjunctival/perilesional 5-fluorouracil injections" (doi: 10.2147/DDDT.S191161). ${ }^{8}$

\section{Author Contributions}

All authors made substantial contributions to conception and design, acquisition of data, or analysis and interpretation of data; took part in drafting the article or revising it critically for important intellectual content; agreed to submit to the current journal; gave final approval of the version to be published; and agree to be accountable for all aspects of the work.

\section{Funding}

Natural Science Foundation of Liaoning Province (No.20170541041). The funders had no role in the study design, in data collection, analysis, or interpretation, in the decision to publish, or in preparation of the manuscript.

\section{Disclosure}

The authors report no conflicts of interest in this work.

\section{References}

1. Lee GA, Hirst LW. Ocular surface squamous neoplasia. Surv Ophthalmol. 1995;39:429-450. doi:10.1016/S0039-6257(05)80054-2

2. Gichuhi S, Sagoo MS, Weiss HA, Burton MJ. Epidemiology of ocular surface squamous neoplasia in Africa. Trop Med Int Health. 2013;18:1424-1443. doi:10.1111/tmi.12203

3. Yousef YA, Finger PT. Squamous carcinoma and dysplasia of the conjunctiva and cornea: an analysis of 101 cases. Ophthalmology. 2012;119:233-240. doi:10.1016/j.ophtha.2011.08.005

4. Atallah M, Joag M, Galor A, et al. Role of high resolution optical coherence tomography in diagnosing ocular surface squamous neoplasia with coexisting ocular surface diseases. Ocul Surf. 2017;15:688-695. doi:10.1016/j.jtos.2017.03.003

5. Zarei-Ghanavati M, Mousavi E, Nabavi A, et al. Changes in in vivo confocal microscopic findings of ocular surface squamous neoplasia during treatment with topical interferon alfa-2b. Ocul Surf. 2018;16:235-241. doi:10.1016/j.jtos.2017.12.003

6. Habibalahi A, Bala C, Allende A, Anwer AG, Goldys EM. Novel automated non invasive detection of ocular surface squamous neoplasia using multispectral autofluorescence imaging. Ocul Surf. 2019;17:540-550. doi:10.1016/j.jtos.2019.03.003

7. Sayed-Ahmed IO, Palioura S, Galor A, Karp CL. Diagnosis and medical management of ocular surface squamous neoplasia. Expert Rev Ophthalmol. 2017;12:11-19. doi:10.1080/17469899.20 17.1263567

8. Sun Y, Hua R. Ocular surface squamous neoplasia: angiographic characteristics and response to subconjunctival/perilesional 5-fluorouracil injections. Drug Des Devel Ther. 2019;13:1323-1334. doi:10.2147/DDDT.S191161

9. Liu Z, Karp CL, Galor A, Al Bayyat GJ, Jiang H, Wang J. Role of optical coherence tomography angiography in the characterization of vascular network patterns of ocular surface squamous neoplasia. Ocul Surf. 2020;18:926-935. doi:10.1016/j.jtos.2020.03.009
10. Theotoka D, Morkin MI, Naranjo A, Dubovy SR, Karp CL. Spontaneous regression of ocular surface squamous neoplasia: possible etiologic mechanisms in cancer resolution. Ocul Surf. 2020;18:351-353. doi:10.1016/j.jtos.2020.03.001

11. Parrozzani R, Frizziero L, Trainiti S, et al. Topical 1\% 5-fluoruracil as a sole treatment of corneoconjunctival ocular surface squamous neoplasia: long-term study. Br J Ophthalmol. 2017;101:1094-1099. doi:10.1136/bjophthalmol-2016-309219

12. Cicinelli MV, Marchese A, Bandello F, Modorati G. Clinical management of ocular surface squamous neoplasia: a review of the current evidence. Ophthalmol Ther. 2018;7:247-262. doi:10.1007/s40123018-0140-z

13. Singh S, Mittal R, Ghosh A, Tripathy D, Rath S. High-resolution anterior segment optical coherence tomography in intraepithelial versus invasive ocular surface squamous neoplasia. Cornea. 2018;37:1292-1298. doi:10.1097/ICO.0000000000001680

14. Joag MG, Sise A, Murillo JC, et al. Topical 5 -fluorouracil $1 \%$ as primary treatment for ocular surface squamous neoplasia. Ophthalmology. 2016;123:1442-1448. doi:10.1016/j. ophtha.2016.02.034

15. Gichuhi S, Macharia E, Kabiru J, et al. Topical fluorouracil after surgery for ocular surface squamous neoplasia in Kenya: a randomised, double-blind, placebo-controlled trial. Lancet Glob Health. 2016;4:e378-e385. doi:10.1016/S2214-109X(16)30052-3

16. Elhamaky TR, Elbarky AM. AS-OCT guided treatment of diffuse conjunctival squamous cell carcinoma with resection, amniotic membrane graft and topical mitomycin C. Clin Ophthalmol. 2019;13:2269. doi:10.2147/OPTH.S229399

17. Al Bayyat G, Arreaza-Kaufman D, Venkateswaran N, Galor A, Karp CL. Update on pharmacotherapy for ocular surface squamous neoplasia. Eye Vis. 2019;6:1-2. doi:10.1186/s40662-019-0150-5

18. Sepulveda R, Pe'er J, Midena E, Seregard S, Dua HS, Singh AD. Topical chemotherapy for ocularsurface squamous neoplasia: current status. Br J Ophthalmol. 2010;94:532-535.

19. Nanji AA, Sayyad FE, Karp CL. Topical chemotherapy for ocular surface squamous neoplasia. Curr Opin Ophthalmol. 2013;24:336-342. doi:10.1097/ICU.0b013e3283622a13

20. Karp CL, Galor A, Chhabra S, Barnes SD, Alfonso EC. Subconjunctival/perilesional recombinant interferon $\alpha 2 b$ for ocular surface squamous neoplasia: a 10-year review. Ophthalmology. 2010;117:2241-2246. doi:10.1016/j.ophtha.2010.03.052

21. Reynolds JE, Martindale W. Martindale: The Extra Pharmacopoeia. 31st ed. London: Royal Pharmaceutical Society; 1996:572-575.

22. Venkateswaran N, Mercado C, Galor A, Karp CL. Comparison of topical 5-fluorouracil and interferon alfa- $2 \mathrm{~b}$ as primary treatment modalities for ocular surface squamous neoplasia. $\mathrm{Am}$ J Ophthalmol. 2019;199:216-222. doi:10.1016/j.ajo.2018.11.007

23. Thomas BJ, Galor A, Nanji AA, et al. Ultra high-resolution anterior segment optical coherence tomography in the diagnosis and management of ocular surface squamous neoplasia. Ocul Surf. 2014;12:46-58. doi:10.1016/j.jtos.2013.11.001

24. Ong SS, Vora GK, Gupta PK. Anterior segment imaging in ocular surface squamous neoplasia. $J$ Ophthalmol. 2016;5435092. doi:10.1155/2016/5435092

25. Chaugule SS, Park J, Finger PT. Topical chemotherapy for giant ocular surface squamous neoplasia of the conjunctiva and cornea: is surgery necessary? Indian $J$ Ophthalmol. 2018;66:55-60. doi:10.4103/ijo.IJO_590_17

26. Miyashita H, Tagawa S, Takeda H, Higashide T, Sugiyama K. Comparison between stereo angiographic findings and histopathologic findings in endotoxin-induced uveitis. Curr Eye Res. 2009;34:536-542. doi:10.1080/02713680902936163 


\section{Publish your work in this journal}

Drug Design, Development and Therapy is an international, peerreviewed open-access journal that spans the spectrum of drug design and development through to clinical applications. Clinical outcomes, patient safety, and programs for the development and effective, safe, and sustained use of medicines are a feature of the journal, which has also been accepted for indexing on PubMed Central. The manuscript management system is completely online and includes a very quick and fair peer-review system, which is all easy to use. Visit http://www. dovepress.com/testimonials.php to read real quotes from published authors. 